RAIRO Operations Research

RAIRO Oper. Res. 37 (2003) 325-335

DOI: $10.1051 /$ ro:2004005

\title{
ÉVALUATION DE LA FIABILITÉ D'UN SYSTÈME DE PRÉVISION DE LA DEMANDE DANS LE CAS D'UNE POLITIQUE DE REGROUPEMENT DES BESOINS
}

\author{
Alice Yalaoui $^{1}$, Éric Châtelet $^{1}$ et Chengbin $\mathrm{Chu}^{2}$
}

\begin{abstract}
To establish production plans or replenishment plans, firms use forecasting systems. Such a system is composed of a forecasting method and a lot-sizing method. We propose a scheme to evaluate the reliability of such a system. This evaluation is based on the impact of demand randomness on the long run total cost. The scheme is illustrated with a system including an exponential smoothing technique for the demand forcasting and the Florian and Klein [5] method for the lot-sizing. Simulation results show that such a system is quite reliable.

Résumé. Pour établir des plans de production et d'approvisionnements, une entreprise utilise un système de prévisions. Celui-ci est constitué d'une méthode de prévision de la demande et d'une méthode de lot-sizing permettant l'obtention de plans prévisionnels. Nous proposons une démarche permettant d'évaluer la fiabilité des prévisions fournies par un tel système. Cette analyse se base sur l'étude de l'impact des aléas de la demande sur les coûts induits par les plans prévisionnels. Nous avons appliqué ce protocole à un système de prévisions constitué du lissage exponentiel et de la méthode de Florian et Klein [5]. Dans les conditions expérimentales considérées, ce système de prévision fournit des informations fiables.
\end{abstract}

Mots Clés. Prévisions, lot-sizing, simulation, fiabilité.

\footnotetext{
${ }^{1}$ UTT LM2S, 12 rue Marie Curie, BP 2060, 10010 Troyes Cedex, France; e-mail : (chatelet, yalaouia) @utt.fr

${ }^{2}$ UTT, LOSI, 12 rue Marie Curie, BP 2060, 10010 Troyes Cedex, France;

e-mail : chu@utt.fr
} 


\section{INTRODUCTION}

\subsection{Problématique}

Le système de production d'une entreprise peut être décomposé en plusieurs sous-systèmes, caractérisés par leur capacité à réaliser un ensemble d'opérations. Des matières premières et des composants circulent à travers ceux-ci pour être assemblés et/ou transformés afin d'obtenir un produit final. De manière à planifier l'ensemble des opérations qui sont liées à ses activités pour chaque élément du système, l'entreprise cherche à établir des plans prévisionnels d'approvisionnements et de production. Il faut donc pour cela estimer les commandes pour les périodes à venir et répartir la charge de travail sur chaque sous-système. L'estimation des besoins est réalisée grâce à des méthodes de prévisions de la demande. À partir de ces informations et pour planifier son activité, l'entreprise cherche alors à minimiser les coûts liés aux plans qu'elle veut établir, c'est-à-dire les coûts fixes de lancement en production ou de commande, les coûts unitaires de production ou d'achat et les coûts de stockage. Elle doit également considérer les capacités de production qui peuvent varier d'un poste à l'autre et dans le temps. Pour résoudre ce problème, des méthodes de regroupement des besoins en lots ont été développées. Elles sont également appelées méthodes de lot-sizing. La planification des activités de l'entreprise est donc basée sur les informations fournies par un système de prévision. Celui-ci est constitué d'une part, d'une méthode de prévision de la demande et d'autre part, d'une méthode de lot-sizing permettant d'obtenir, à partir des prévisions de la demande des plans de production ou d'approvisionnement prévisionnels.

À partir d'un historique de la demande, les méthodes de prévision permettent d'estimer la demande pour les périodes à venir, mais ne peuvent pas anticiper la composante aléatoire de la demande. En effet, on peut constater a posteriori des écarts entre les prévisions et les réalisations de la demande pour une même période. De ce fait, toute entreprise aspire à la mise en place ou à la possession d'outils de prévisions les plus fiables possibles afin d'établir des plans de production prévisionnels qui permettent de répondre le plus justement à la demande.

\subsection{LES MÉTHODES DE LOT-SIZING}

Drexl et Kimms [3] ou Kuik et al. [11] ont passé en revue les différents modèles de lot-sizing. Ces auteurs proposent des classifications des méthodes en fonction des problèmes auxquels elles s'appliquent. Parmi la grande diversité des modèles existants, nous nous intéressons ici au cas d'un produit devant passer sur une ressource. Dans le cas où cette ressource est de capacité infinie, avec autorisation de pénuries (incapacité à répondre à la demande en temps voulu, générant un coût supplémentaire dû à la perte de la commande ou au retard à la livraison), le problème peut être formulé de la manière suivante, pour un horizon de $T$ périodes : 


$$
\begin{gathered}
\text { (P1) } \operatorname{Min}\left[\sum_{i=1}^{T} p_{i}\left(x_{i}\right)+h_{i}\left(I_{i}\right)\right] \\
\sum_{i=1}^{T} x_{i}=\sum_{i=1}^{T} d_{i} \\
x_{i} \geq 0 \quad \forall i 1 \leq i \leq T \\
\text { avec } I_{i}=\sum_{j=1}^{i}\left(x_{j}-d_{j}\right) \quad \forall i 1 \leq i \leq T .
\end{gathered}
$$

À la période $i, I_{i}$ est le niveau de stock, $x_{i}$ est la quantité produite, $d_{i}$ la demande, $p_{i}$ et $h_{i}$ sont respectivement les coûts de production (incluant le coût de lancement) et de stockage. La solution optimale de ce problème est donnée par la méthode de Wagner-Whitin [14]. Dupont [4] présente d'autres méthodes moins coûteuses en temps de calcul pour ce problème. Citons par exemple les heuristiques Least Unit Cost, Part period algorithm, ou l'algorithme de Silver-Meal.

Si l'on rajoute une contrainte de capacité à la ressource considérée pour le cas mono-produit, le problème appartient alors à la famille du Capacitated Lot Sizing Problem (CLSP) étudiée par Bitran et Yanasse [2]. La capacité maximale de production à la période $i$ est notée $C_{i}$. Une formulation du CLSP est donnée par le modèle (P2).

$$
\begin{gathered}
\text { (P2) } \operatorname{Min}\left[\sum_{i=1}^{T} p_{i}\left(x_{i}\right)+h_{i}\left(I_{i}\right)\right] \\
0 \leq x_{i} \leq C_{i} \quad \forall i \quad 1 \leq i \leq T \\
\sum_{i=1}^{T} x_{i}=\sum_{i=1}^{T} d_{i} \\
I_{0}=I_{T}=0
\end{gathered}
$$

et on suppose que $\sum_{i=1}^{T} C_{i} \geq \sum_{i=1}^{T} d_{i}$.

Florian et Klein [5] ont étudié ce problème dans le cas où la capacité maximale disponible par période est constante sur l'horizon de travail $\left(C_{i}=C, 1 \leq i \leq T\right)$. Les fonctions $p_{i}$ et $h_{i}$ associées aux coûts de production et de possession sont supposées concaves. La méthode exacte qu'ils proposent est en $O\left(T^{4}\right)$, c'est-à-dire qu'elle nécessite un temps de calcul théorique de l'ordre de $T^{4}$. Elle est composée de deux étapes : la résolution des sous-problèmes d'une part et la résolution globale d'autre part. La résolution d'un sous-problème $(u, v)$ est basée sur la propriété de décomposition du problème établie par les auteurs, et consiste à trouver le plus court chemin pour aller de la période $u$ à la période $v$, avec $1 \leq u<v \leq T$.

D'autres travaux sont dédiés à des problèmes proches de celui-ci. Par exemple, Lambert et Luss (1981) et Kirca (1990) se sont intéressés au cas où les capacités varient d'une période à l'autre. Les travaux de Florian et Klein ont été repris par Jagannathan et Rao (1973) qui utilisent les propriétés de Florian et Klein pour 
développer une méthode heuristique adaptée au cas où les fonctions de coût sont quelconques [7]. En effet, Florian et al. [6] montrent que ce problème est NPdifficile dans ce cas, c'est-à-dire qu'il est peut probable qu'il existe un algorithme de complexité polynomiale pour le résourdre de manière exacte [6]. Citons également les travaux plus récents de Van Hoesel et Wagelmans qui supposent que la fonction de coût de stockage est linéaire [13]. Ils apportent alors une modification à l'algorithme de Florian et Klein [5] permettant d'obtenir la solution exacte du problème en $O\left(T^{3}\right)$.

\section{3. Évaluation DES PERFORMANCES DES MÉTHOdES DE LOT-SIZING}

Jeunet [8] cite de nombreux travaux réalisés sur l'évaluation de la performance des méthodes de lot-sizing dans le cas mono-produit : Blackburn et Millen (1979), Colerman et McKnew (1990), Zhu et al. (1994), etc. [8]. Dans la plupart des cas, on retrouve deux principaux critères d'évaluation. Le premier est la performance en terme de coût dans le cas d'une méthode heuristique, c'est-à-dire l'écart entre le coût obtenu par la méthode étudiée et le coût optimal. Le second critère est le temps de calcul qui reflète la complexité de la méthode. Citons par exemple les travaux de Saydam et Evans [12] qui portent sur le calcul des performances de plusieurs heuristiques par rapport à la méthode de Wagner-Whitin [14], en se basant sur ces deux critères [12]. Mais ceux-ci ignorent l'environnement perturbé dans lequel sont utilisées ces méthodes. Afin d'y remédier, en 2000, Jeunet et Jonard ont étudié comment réagissent certaines techniques de lot-sizing face à une demande qui fluctue [9]. Pour cela, ils introduisent une mesure de la robustesse des algorithmes. Il s'agit d'une mesure du degré de stabilité des ordres planifiés par un algorithme donné, en réponse à des changements de la demande estimée. Ils examinent plus particulièrement comment les variations de la demande affectent la planification de la production dans le cas d'un produit passant sur une ressource de capacité illimitée.

Du fait de la grande diversité des problèmes de lot-sizing existants [11], il existe d'autres études de performances, adaptées aux différents contextes de ces problèmes. Par exemple, Johansen [10] a comparé les méthodes pour une demande déterministe avec celles pour une demande stochastique. Il montre que les techniques supposant l'information déterministe sont plus efficaces si l'incertitude sur la demande est relativement faible. L'avantage de ces techniques diminue avec l'augmentation de l'incertitude. Citons également les travaux de Jönsen et Silver (1985) et Kamarkar et al. (1992) en environnement incertain et ceux de Maes et Van Wassenhove (1986) sur l'étude des différentes heuristiques dans le cas multiproduits sur une machine [11].

Nous nous intéressons ici au problème de lot-sizing à un produit et une ressource, mais à la différence de Jeunet et Jonard [9], nous considérons le cas où la ressource est de capacité limitée. Nous étudions ici un système de prévision constitué d'une méthode de prévision de la demande et d'une méthode de lot-sizing. Nous cherchons à évaluer la qualité d'un tel système face à l'introduction d'aléas au niveau de la demande. Nous allons dans le second paragraphe aborder la démarche 
d'évaluation de la fiabilité des prévisions que nous proposons à travers un exemple de système de prévisions. Celui-ci est constitué du lissage exponentiel associé à la méthode de Florian et Klein [5]. Dans un troisième paragraphe, nous présentons les principaux résultats et nous terminerons par les conclusions et perspectives.

\section{DÉmarche}

Nous cherchons à évaluer la fiabilité des informations fournies par un système de prévision. Cette étude est basée sur l'analyse de l'impact des aléas de la demande sur le coût obtenu, pour un horizon donné, par la méthode de lot-sizing. Ainsi, nous pourrons quantifier l'effet des aléas de la demande sur le système de production tributaire de ces prévisions. D'une manière générale, afin de pouvoir étudier la fiabilité du système, il nous faut définir un critère d'évaluation. On étudie alors le comportement de ce critère en fonction du niveau du phénomène étudié, qui peut être contrôlable ou non. Le système peut alors être considéré comme fiable si le critère d'évaluation est dans une zone de tolérance définie pour les perturbations considérées. Nous allons revenir sur les caractéristiques du système étudié avant de détailler les trois étapes de la démarche d'évaluation de la fiabilité des prévisions.

\subsection{LE SYSTÈME DE PRÉVISION}

Considérons tout d'abord un historique de la demande de longueur $L$. Selon Baglin et al. en 2001, différents éléments interviennent de manière générale dans la loi suivie par la demande [1]. Celle-ci est en effet composée d'un niveau initial $a$, d'une tendance $b$, d'une saisonnalité sur un cycle de $S$ périodes où à chaque période est affecté un coefficient de saisonnalité $C S_{j}(1 \leq j \leq S)$, et de résidus ou aléas $R_{t}(1 \leq t \leq L)$. Nous supposons que ces aléas suivent une loi normale centrée et d'écart type $\sigma_{R}$ notée $\mathrm{N}\left(0, \sigma_{R}\right)$. Le comportement de la demande à la période $t(1 \leq t \leq L)$ notée $d_{t}$ peut alors être modélisé de la manière suivante :

$$
d_{t}=a+b+C S_{\bmod (t / S)}+R_{t} \quad \forall \quad 1 \leq t \leq L
$$

où $\bmod (t / S)$ est le reste de la division de $t$ par $S$. Sur l'historique $L$, les réalisations de la demande $d_{t}(1 \leq t \leq L)$ sont connues, mais les valeurs des différents éléments la constituant sont quant à elles inconnues. Considérons maintenant un horizon de planification de $H$ périodes. Nous cherchons à réaliser des prévisions de la demande sur cet horizon. Notons $\hat{d}_{t}^{\tau}$ l'estimation de la demande réalisée à la l'instant $\tau$ $(1 \leq \tau \leq H)$ pour la période $t(\tau<t \leq H)$. Pour pouvoir réaliser de telles prévisions de la demande, il faut tout d'abord estimer la loi que celle-ci suit, soit les valeurs prises par les différents éléments qui la constituent. Notons respectivement $\hat{a}, \hat{b}$ et $\hat{C} S_{j}(1 \leq j \leq S)$ les estimations du niveau initial, de la tendance et des coefficients de saisonnalité, réalisées à partir de l'historique $L$.

Le lissage exponentiel introduit par Winters (1960) permet d'estimer, à partir de l'historique $L$, les différentes composantes de la demande quelles qu'en soient les caractéristiques (présence ou non de tendance et/ou saisonnalité) [15]. Ces 
estimations sont réalisées par l'intermédiaire de trois coefficients de lissage notés $\alpha, \beta$ et $\gamma(0 \leq \alpha, \beta, \gamma \leq 1)$ en réalisant des suites de moyennes pondérées et en affectant à chaque donnée un poids qui décroît exponentiellement avec l'âge. Cette méthode de prévision de la demande est l'une des plus flexibles et réactives [1]. Le modèle obtenu ci-dessous est utilisé pour réaliser des prévisions de la demande à un instant $\tau(1 \leq \tau \leq H)$ pour une période $t$ de l'horizon $H$.

$$
\hat{d}_{t}^{\tau}=\hat{a}+\hat{b}+\hat{C} S_{\bmod (t / S)} \quad \forall \quad 1 \leq \tau \leq H \quad \text { et } \forall \quad \tau<t \leq H .
$$

Supposons que l'on choisisse de travailler selon le principe d'un plan glissant sur l'horizon $H$, comme ce qui se passe dans la pratique. Soit un nombre $T$ de périodes tel que $0<T \leq H$. À un instant $\tau(1 \leq \tau \leq H)$, on réalise alors des prévisions de la demande pour les $T$ périodes à venir. La méthode de lot-sizing de Florian et Klein [5] permet, à partir de ces prévisions d'obtenir le plan de production sur l'horizon $T$ d'un produit devant passer par une ressource de capacité limite $C$ constante. La solution est optimale dans le cas où les fonctions associées aux coûts de production et de possession sont concaves. Les situations de pénurie sont permises.

À partir d'un historique de $L$ périodes, ce système de prévision réalise donc un modèle de la loi suivie par la demande et ce modèle permet de réaliser des estimations de la demande pour les $T$ périodes à venir. Ces prévisions sont alors les données d'entrée de la méthode de Florian et Klein qui établit le plan de production qui minimise la somme des coûts sur l'horizon $T$.

\subsection{Critère D'Évaluation De la fiabilité DU Système}

Afin de pouvoir quantifier l'effet des aléas de la demande sur le système de production, le critère que nous avons choisi dépend de l'écart type $\sigma_{R}$ de la loi suivie par la demande. Pour une valeur de l'écart type donnée, on définit deux coûts. Le premier est le coût réel noté $C_{1}$ observé lorsque le système fonctionne à partir des prévisions de la demande sur l'horizon de $H$ périodes. $C_{1}$ est la somme des coûts de production et de possession sur $H$. Le second coût noté $C_{2}$ est quant à lui calculé a posteriori. Une fois les $H$ périodes écoulées, les réalisations de la demande par période sont connues et l'on peut calculer ce coût minimal sur les $H$ périodes. Le critère d'évaluation des performances du système de prévisions est alors :

$$
\text { critère }\left(\sigma_{R}\right)=\frac{C_{1}}{C_{2}}\left(\sigma_{R}\right)
$$

\subsection{MÉthode D'Évaluation Du CRITÈre POUR Un ÉCART TYPE FIXÉ}

Afin d'évaluer le critère de performance tel que nous venons de le définir, la démarche proposée peut être décomposée en trois étapes présentées ci-dessous. 


\subsubsection{Première étape : génération des données}

Pour un écart type fixé de la loi des aléas, il faut commencer par générer les données du problème. On dispose pour cela d'un modèle de la demande avec des valeurs fixées arbitrairement pour les différentes composantes :

$$
d_{t}=a+b+C S_{\bmod (t / S)}+R_{t} \text { avec } R \sim>N\left(0, \sigma_{R}\right) \text { et } 1 \leq t \leq L+H .
$$

On génère alors en utilisant ce modèle la demande sur un historique de $L$ périodes et sur un horizon de planification de $H$ périodes.

\subsubsection{Deuxième étape : optimisation du lissage exponentiel}

Le lissage exponentiel utilise trois coefficients $\alpha, \beta$ et $\gamma$ tels que $0 \leq \alpha, \beta, \gamma \leq 1$. Il faut déterminer les valeurs à affecter à ces coefficients de manière à ajuster le modèle. Pour ce faire, il faut rechercher la combinaison des coefficients du lissage qui minimise la moyenne et l'écart type des erreurs entre les réalisations de la demande sur l'historique et les valeurs obtenues par le lissage sur celui-ci. Cette recherche peut être effectuée par énumération, en incrémentant les valeurs de $\alpha, \beta$ et $\gamma$ d'un pas de 0,1 à partir de 0 et ce jusqu'à 1 . On obtient alors les estimations $\hat{a}, \hat{b}$ et $\hat{C} S_{j}(1 \leq j \leq S)$ des composantes de la demande.

\subsubsection{Troisième étape : évaluation de l'impact de l'écart type de la loi suivie par les aléas sur la qualité des prévisions}

Afin de calculer le coût réel $C_{1}$, nous travaillons en horizon glissant sur les $H$ périodes de planification. Le mode d'évaluation de ce coût est schématisé à la figure 1. Soit $\tau$ la période courante $(\tau=1, \ldots, H)$. On se place au début de l'horizon $H(\tau=1)$, le coût $C_{1}$ étant initialisé à zéro et les deux premières étapes de la méthode ont été effectuées.

La demande $d_{\tau}$ sera connue une fois la période écoulée. On utilise donc le modèle obtenu par le lissage exponentiel à partir de l'historique $L$ pour réaliser des prévisions sur l'intervalle de temps $[\tau, \ldots, \tau+T](\tau+T \leq H)$. On rappelle que le nombre $T$ représente l'horizon choisi par l'entreprise pour réaliser à un moment donné ses prévisions et le nombre $H$ représente l'horizon glissant que nous choisissons pour calculer le critère.

Les prévisions obtenues sont alors utilisées par la méthode de Florian et Klein et l'on obtient un plan prévisionnel pour l'horizon $T$. On considère alors que la période courante $\tau$ s'écoule et que l'on prend connaissance de la demande réelle $d_{\tau}$ pour cette période. Cette demande désormais connue de la période $\tau$ qui vient de s'écouler est utilisée pour ajuster au mieux le modèle du comportement de la demande. On réactualise donc les estimateurs des composantes de la demande (calcul des coefficients). Lorsque $\tau=H$, on calcule $C_{1}$, le coût associé aux plans obtenus successivement à chaque itération $\tau$ et établis sur la base des prévisions.

Il reste à calculer le coût minimal de référence $C_{2}$, associé aux approvisionnements résultants de l'application du modèle de Florian et Klein à l'ensemble des demandes connues. On peut alors, pour l'écart type $\sigma_{R}$ fixé, évaluer $C_{1} / C_{2}$. 


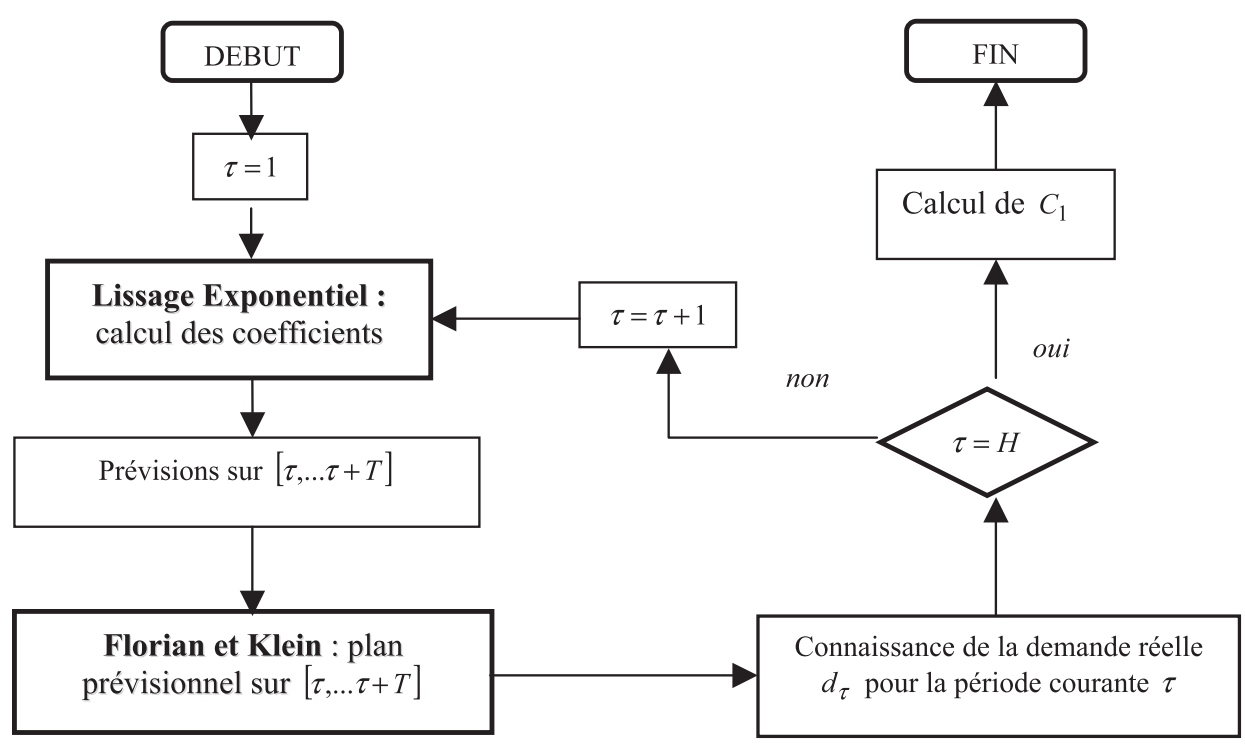

Figure 1. Procédure d'évaluation de $C_{1}$.

\section{4. ÉVALUATION DU CRITÈRE POUR PLUSIEURS ÉCARTS TYPES}

Nous venons de voir le mode d'évaluation des deux coûts pour un écart type fixé. Afin de pouvoir évaluer le comportement du système de manière générale face à des aléas de la demande, il faut répéter cette opération pour différentes valeurs de $\sigma_{R}$. La démarche générale d'évaluation de la fiabilité peut alors être synthétisée comme sur la figure 2 .

On commence avec un écart type nul (pas d'aléas de la demande) et l'on se fixe une borne supérieure arbitraire notée $n$ sur l'écart type ainsi qu'un pas $p$ pour l'incrémentation. On réalise donc les trois étapes en boucle jusqu'à ce que l'on arrive à $n$. On peut alors au fur et à mesure représenter l'évolution du rapport des coûts en fonction de l'écart type.

\section{RÉSUltats}

Afin de réaliser l'évaluation de la fiabilité du système de prévision constitué du lissage exponentiel et de la méthode de Florian et Klein, nous avons généré 30 types de problèmes avec des caractéristiques différentes, c'est-à-dire différentes valeurs affectées aux composantes de la demande décrites dans la section 2.1. Nous avons utilisé des générateurs de lois uniformes pour les différents coefficients $a, b, S, C, C S$ et $T$ avec respectivement les intervalles [10, 100], $\left[10^{-5}, 10^{-1}\right],[2,12]$, $[20,150],[-1,5,1,5]$ et $[4,10]$. Nous avons repris le générateur des coûts de Florian 


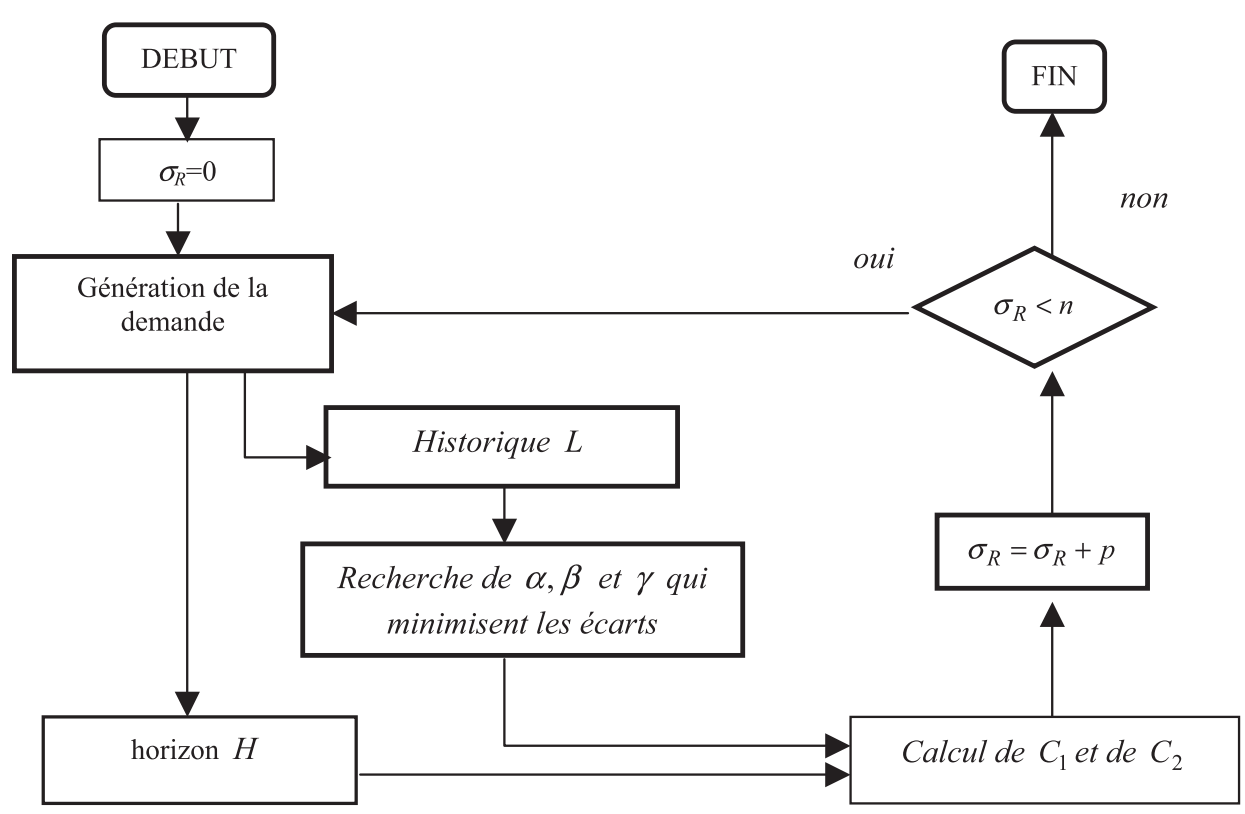

Figure 2. Démarche générale.

et Klein. La fonction coût de production est obtenue avec l'expression suivante :

$$
p_{i}\left(x_{i}\right)=s \times \delta\left(x_{i}\right)+p \times x_{i} \text { avec } 1 \leq i \leq H
$$

avec $\delta\left(x_{i}\right)=1$ si $x_{i}>0$ et $\delta\left(x_{i}\right)=0$ sinon. Les paramètres $s$ et $p$ sont le coût de lancement et le coût unitaire de production. En ce qui concerne la fonction coût de possession, son expression générale est la suivante :

$$
h_{i}\left(I_{i}\right)=\max \left(0, h_{1} I_{i}\right)+\max \left(0,-h_{2} I_{i}\right) .
$$

Tous les coûts $s, p, h_{1}$ et $h_{2}$ sont générés uniformément dans l'intervalle [1, 30]. En ce qui concerne la taille de l'horizon glissant $H$, plusieurs longueurs $(100,500$, 1000) ont été testées. Nous avons pu observer que la taille de $H$ n'influe pas sur la qualité des résultats et nous avons préféré finalement garder une taille $H=100$, le temps de calcul de $C_{2}$ devenant trop important pour des valeurs plus grandes.

Le comportement du rapport $C_{1} / C_{2}$ a été étudié pour chacun des problèmes générés. Le système de prévision pourra alors être qualifié de fiable si le critère présente une stabilité quand à l'introduction des aléas et s'il ne dépasse pas les limites de tolérances fixées par l'entreprise. Pour tous les problèmes générés, l'évolution du critère est semblable à celle présentée à la figure 3 .

Il apparaît, pour tous les problèmes générés, que le rapport des coûts augmente linéairement avec l'écart type $\sigma_{R}$. Son évolution semble fortement corrélée (coefficient de corrélation $R^{2} \approx 0.99$, Fig. 3 ) avec celle de ce dernier. L'augmentation 


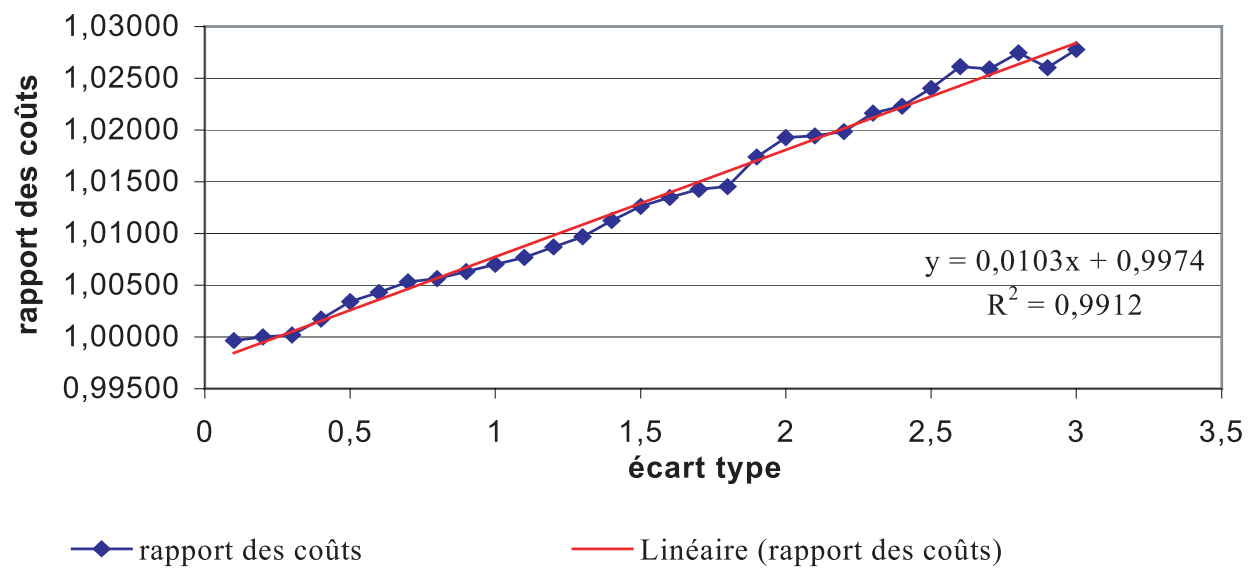

Figure 3. Évolution de $C 1 / C 2$ en fonction de $\sigma_{R}$.

moyenne du critère est stable et est d'une unité lorsque l'écart type augmente de cent unités. Bien que nous ne nous soyons pas fixés de limites pour la zone de tolérance du critère a priori, nous pouvons dire, grâce au protocole expérimental, que le lissage exponentiel, associé ici à la méthode de Florian et Klein est un système de prévision fiable, pour les conditions expérimentales préalablement fixées. Pour chaque problème généré, différentes valeurs de $T$ ont été testées et nous avons pu observer que le nombre de périodes $T$ choisi pour effectuer les prévisions n'a pas d'influence sur le coût $C_{1}$. Un nombre minimum de période à considérer peut toutefois être déterminé de manière à ne pas avoir de problèmes non réalisables, en fonction de la capacité de production et des caractéristiques de la demande.

Ces simulations ont été réalisées sur un Pentium III 700. Nous avons pu constater que le temps de calcul du coût $C_{1}$ augmente avec le nombre de périodes $T$ choisies pour établir les plans prévisionnels alors que celui de $C_{2}$ est quant à lui stable. Par exemple, pour un horizon glissant $H$ de 100 périodes, le temps moyen de calcul de $C_{2}$ est de $21 \mathrm{~s}$ CPU.

\section{Conclusions et Perspectives}

Nous avons proposé une nouvelle démarche d'évaluation de la fiabilité d'un système de prévisions, constituée d'une méthode de prévision de la demande et d'une méthode de lot-sizing. Afin d'illustrer ce protocole d'évaluation des performances, nous avons choisi de l'appliquer ici au lissage exponentiel associé à la méthode de Florian et Klein [5]. Dans les conditions expérimentales choisies, c'est-à-dire par rapport à la nature des aléas retenue, les informations fournies par ce système de prévision sont fiables. 
Cette démarche peut être appliquée à d'autres systèmes de prévisions. Elle peut être utilisée pour comparer différents systèmes de prévision dans différentes situations. D'autres indicateurs peuvent être également utilisés, comme l'étude de la stabilité des solutions suite à l'introduction d'aléas. D'autre part, nous n'avons considéré dans ce travail qu'un seul phénomène aléatoire, suivant une loi symétrique, qui peut intervenir sur un système de production. D'autres types d'aléas suivant des lois différentes (asymétriques par exemple) peuvent être considérés comme les défaillances des ressources du système de production.

\section{RÉFÉRENCES}

[1] G. Baglin, O. Bruel, A. Garreau, M. Greif et C. van Delft, Management Industriel et logistique. $3^{e}$ édition, Gestion Economica (2001).

[2] G.R. Bitran and H.H. Yanasse, Computational complexity of the capacitated lot-size problem. Manage. Sci. 28 (1982) 1174-1186.

[3] A. Drexl and A. Kimms, Lot sizing and scheduling - Survey and extensions. Eur. J. Oper. Res. 99 (1997) 221-235.

[4] L. Dupont, La gestion industrielle. Paris, Hermes (1998).

[5] M. Florian and M. Klein, Deterministic production planning with concave with concave costs and capacity constraints. Manage. Sci. 18 (1971) 12-20.

[6] M. Florian, J.K. Lenstra and A.H.G. Rinnooy Kan, Deterministic production Planning Algorithms and Complexity. Manage. Sci. 26 (1980) 669-679.

[7] R. Jagannathan and M.R.A. Rao, Class of deterministic producting planning problems. Manage. Sci. 19 (1973) 1295-1300.

[8] J. Jeunet, Modèles d'approvisionnement du type MRP : évaluation et robustesse. Thèse, Université de Strasbourg I, France (1997).

[9] J. Jeunet and N. Jonard, Measuring the performance of lot-sizing techniques in uncertain environments. Int. J. Prod. Econom. 64 (2000) 197-208.

[10] S.G. Johansen, Lot sizing for varying degrees of demand uncertainty. Int. J. Prod. Econom. 59 (1999) 405-414.

[11] R. Kuik, M. Salomon and L.N. van Wassenhove, Batching decisions : structure and models. Eur. J. Oper. Res. 75 (1994) 243-263.

[12] C. Saydam and J.R. Evans, A comparative performance analysis of the Wagner-Within algorithm and lot-sizing heuristics. Comput. Ind. Engng. 18 (1990) 91-93.

[13] C.P.M. Van Hoesel and A.P.M. Wagelmans, $\mathrm{An} \mathrm{O}\left(\mathrm{T}^{3}\right)$ algorithm for the economic Lot-sizing Problem with Constant Capacity. Manage. Sci. 42 (1996) 142-150.

[14] H.M. Wagner and T.M. Whitin, Dynamic Version of the economic lot-size Modem. Manage. Sci. 5 (1958) 89-96.

[15] P.R. Winters, Forecasting Sales by Exponentally Weighted Moving Averages. Manage. Sci. 6 (1960) 324-342. 